\title{
Raman lasing and soliton mode-locking in lithium niobate microresonators
}

\author{
Mengjie Yu', Yoshitomo Okawachi², Rebecca Cheng ${ }^{1}$, Cheng Wang $\mathbb{C}^{3}$, Mian Zhang ${ }^{4}$, Alexander L. Gaeta $\mathbb{E}^{2}$ and \\ Marko Lončar ${ }^{1}$
}

\begin{abstract}
The recent advancement in lithium-niobite-on-insulator (LNOI) technology is opening up new opportunities in optoelectronics, as devices with better performance, lower power consumption and a smaller footprint can be realised due to the high optical confinement in the structures. The LNOI platform offers both large $x^{(2)}$ and $x^{(3)}$ nonlinearities along with the power of dispersion engineering, enabling brand new nonlinear photonic devices and applications for the next generation of integrated photonic circuits. However, Raman scattering and its interaction with other nonlinear processes have not been extensively studied in dispersion-engineered LNOI nanodevices. In this work, we characterise the Raman radiation spectra in a monolithic lithium niobate (LN) microresonator via selective excitation of Raman-active phonon modes. The dominant mode for the Raman oscillation is observed in the backward direction for a continuous-wave pump threshold power of $20 \mathrm{~mW}$ with a high differential quantum efficiency of $46 \%$. We explore the effects of Raman scattering on Kerr optical frequency comb generation. We achieve mode-locked states in an X-cut LNOI chip through sufficient suppression of the Raman effect via cavity geometry control. Our analysis of the Raman effect provides guidance for the development of future chip-based photonic devices on the LNOI platform.
\end{abstract}

\section{Introduction}

The monolithic lithium-niobite-on-insulator (LNOI) platform has attracted significant interest for the realisation of next-generation nonlinear photonic devices and the observation of new nonlinear dynamics due to its large $\chi^{(2)}\left(r_{33}=3 \times 10^{-11} \mathrm{~m} / \mathrm{V}\right)$ and $X^{(3)}$ nonlinearities $\left(n_{2}=\right.$ $\left.1.8 \times 10^{-19} \mathrm{~m}^{2} / \mathrm{W}\right)^{1-16}$. The LNOI platform is creating new opportunities for large-scale integration of optical and electronic devices on a single chip, as it combines the material properties of lithium niobate with the integration power of nanophotonics. By leveraging recent advances in the fabrication of ultra-low-loss lithium niobate (LN) nanowaveguides and microring resonators ${ }^{1}$, researchers have demonstrated Kerr optical frequency combs

\footnotetext{
Correspondence: Marko Lončar (loncar@seas.harvard.edu)

${ }^{1}$ Department John A. Paulson School of Engineering and Applied Sciences,

Harvard University, Cambridge, MA 02138, USA

${ }^{2}$ Department of Applied Physics and Applied Mathematics, Columbia

University, New York, NY 10027, USA

Full list of author information is available at the end of the article.

These authors contributed equally: Mengjie Yu, Yoshitomo Okawachi
}

$(\mathrm{OFCs})^{2-4}$, broadband electro-optic combs ${ }^{5}$, highly efficient second harmonic generation ${ }^{6-8}$ and multipleoctave-spanning supercontinuum generation (SCG) ${ }^{8,9}$. $\mathrm{LN}$ is known as a Raman-active crystalline material with several strong vibrational phonon branches in different polarisation configurations ${ }^{17-23}$. There has been evidence of Raman scattering in LN discs or whispering gallery resonators ${ }^{24,25}$ fabricated by mechanical polishing ${ }^{26}$. The Raman effect in integrated photonic devices not only enables Raman lasers for the generation of new frequencies at low optical powers ${ }^{27-34}$ but can also lead to nontrivial nonlinear interactions through tailoring the dispersion properties, such as the interplay between the Raman effect, and both $\chi^{(2)}$ and $\chi^{(3)}$ effects, impacting Kerr comb formation, electro-optic comb formation and supercontinuum generation ${ }^{35-41}$. Recent work by Hansson et al. has shown that aligning the cavity free spectral range (FSR) to the Raman gain can allow for the generation of an octave-spanning Raman frequency comb ${ }^{42}$. The LN photonic circuit is particularly appealing for

\section{(c) The Author(s) 2020}

(c) (i) Open Access This article is licensed under a Creative Commons Attribution 4.0 International License, which permits use, sharing, adaptation, distribution and reproduction cc) in any medium or format, as long as you give appropriate credit to the original author(s) and the source, provide a link to the Creative Commons license, and indicate if changes were made. The images or other third party material in this article are included in the article's Creative Commons license, unless indicated otherwise in a credit line to the material. If material is not included in the article's Creative Commons license and your intended use is not permitted by statutory regulation or exceeds the permitted use, you will need to obtain permission directly from the copyright holder. To view a copy of this license, visit http://creativecommons.org/licenses/by/4.0/. 
microresonator-based Kerr frequency comb generation, since the presence of a large second-order nonlinearity $X^{(2)}$ offers advantageous functionality for a fully on-chip optical clock and metrology, a key element missing from current mature silicon nitride or silica technologies. Due to the large Raman gain in a crystalline material, a strong interplay between Raman scattering and four-wave mixing (FWM) has been observed in materials such as diamond and silicon ${ }^{36}$, and strategies have been proposed to suppress these interactions by controlling the $\mathrm{FSR}^{36,39,43}$. However, Raman scattering and its influence on soliton mode-locking remain largely unexplored in dispersionengineered monolithic LNOI devices.

In this paper, we demonstrate multi-wavelength Raman lasing in an X-cut high- $Q$ LN microresonator with Raman frequency shifts of $250 \mathrm{~cm}^{-1}, 628 \mathrm{~cm}^{-1}$, and $875 \mathrm{~cm}^{-1}$ via pumping with the transverse electric (TE) polarisation and a shift of $238 \mathrm{~cm}^{-1}$ with the traverse magnetic (TM) polarisation. The dominant Raman oscillation occurs in the backward direction with respect to the pump, and the backward Raman gain coefficient is measured to be $1.3 \mathrm{~cm} / \mathrm{GW}$ for the $250 \mathrm{~cm}^{-1}$ Raman shifted line for the TE polarisation and $0.07 \mathrm{~cm} / \mathrm{GW}$ for the $238 \mathrm{~cm}^{-1}$ Raman shifted line for the TM polarisation. In both cases, a $1.5-\mu \mathrm{m}$ pump is used to excite the sample. To our knowledge, this is the first characterisation of multi-wavelength Raman lasing on a monolithic LN chip. In addition, we investigate the effects of the Raman process on Kerr comb generation and soliton mode-locking for both polarisations and show that the Raman effect can be controlled to enable modelocked Kerr comb formation for the TM polarisation.

\section{Results}

$\mathrm{LN}$ is a uniaxial material with its crystal axis along the $\mathrm{z}$ direction, as shown in Fig. 1a. LN devices are fabricated on an X-cut thin-film wafer, where the $x$-axis is normal to the wafer plane. LN has two Raman-active phonon symmetries: the A symmetry polarised along the $z$-axis and the E symmetry polarised in the degenerate $x-y$ plane ${ }^{19,21}$ due to the atomic vibration. Furthermore, both transverse (TO) and longitudinal (LO) optical phonon modes exist. An X-cut wafer allows access to both TO and LO modes in both symmetries. The selection rules of Raman scattering depend on the wavevectors and polarisation of the pump and Stokes fields ${ }^{17-20}$. Two different cavity geometries are used in our experiments (Fig. 1b, c), where the TE-polarised light is mostly parallel to the crystal axis in the racetrack geometry and the TM-polarised light is parallel to the non-polar axis ( $x$-axis).

\section{Characterisation of Raman scattering}

The experimental setup for Raman characterisation is shown in Fig. 1d. We inject an amplified continuous-wave (CW) pump laser centred at $1560 \mathrm{~nm}$ into a monolithically integrated LN racetrack microresonator [Fig. 1b]. The device is cladded with silicon oxide with a top waveguide width of $1.2 \mu \mathrm{m}$ and an etch depth of $450 \mathrm{~nm}$ on an 800$\mathrm{nm}$-thick LN thin film. The racetrack design allows for two long straight waveguide regions to maximise the interaction with the TO optical phonon mode for the TE polarisation. The FSR of the microresonator is $30 \mathrm{GHz}$, which is within the Raman gain bandwidth ${ }^{19}$. The intrinsic $Q$ of the resonator is $\sim 1.5 \times 10^{6}$ for both the TE and TM modes (see Supplementary Information). We record the Raman emission spectra in both the forward and backward directions using an optical circulator and two optical spectrum analysers at various pump powers in the bus waveguide. For the TE polarisation, we observe several Raman oscillations [Fig. 2a] with frequency shifts of $250 \mathrm{~cm}^{-1}(7.5 \mathrm{THz}), \quad 628 \mathrm{~cm}^{-1}$ $(18.8 \mathrm{THz})$, and $875 \mathrm{~cm}^{-1}(26.2 \mathrm{THz})$, corresponding to the optical phonon branches of $\mathrm{A}(\mathrm{TO})_{1}, \mathrm{~A}(\mathrm{TO})_{4}$, and $\mathrm{A}$ (LO) $)_{4}$, respectively. The 1st Stokes peak $\left(250 \mathrm{~cm}^{-1}\right)$ has the lowest pump threshold of $20 \mathrm{~mW}$ with a high differential conversion efficiency of $46 \%$, as shown in Fig. 2b. To our knowledge, this is the highest quantum conversion efficiency reported in an LN material. As the pump power increases, a mini-comb starts to form around the 1st Stokes peak due to the anomalous group velocity dispersion (GVD). This mini-comb prevents the first Stokes line from monotonically increasing with power. In addition, cascaded Raman peaks form $\sim 1691 \mathrm{~nm}$ at $170 \mathrm{~mW}$ of pump power in the forward direction. Notably, strong spectral peaks at $1691 \mathrm{~nm}$ are also observed in the backward direction, largely due to an FWM process where the dominant 1st Stokes line acts as the pump. The 2nd and 3rd Stokes peaks appear as the pump power reaches $200 \mathrm{~mW}$ and $400 \mathrm{~mW}$, respectively. The efficiency of the Raman effect is higher in the backward direction, which is phase-matched ${ }^{44}$; this is particularly true for microscale waveguides that feature a non-negligible longitudinal electric field component ${ }^{45}$. This asymmetric gain can also be attributed to strong polaritonic effects that affect the phase-matching conditions in the forward direction ${ }^{24,46}$. We estimate the Raman gain $g_{\mathrm{R}}$ of the 1 st Stokes line to be $1.3 \mathrm{~cm} / \mathrm{GW}$ based on $^{33}$. For the 2nd and 3rd Stokes peaks, we are unable to extract the Raman gain due to the presence of Kerr and other Raman processes influencing the pump power. Previously, the Raman gain of the corresponding mode in bulk LN was reported to be $12.5 \mathrm{~cm} / \mathrm{GW}$ at $488 \mathrm{~nm}$ by Bache $^{23}, 8.9 \mathrm{~cm} / \mathrm{GW}$ at $694 \mathrm{~nm}$ by Boyd ${ }^{21}$, and $5 \mathrm{~cm} / \mathrm{GW}$ at $1047 \mathrm{~nm}$ by Johnson \& Chunaev et al. ${ }^{22}$, in good agreement with our measurement based on the relation $g_{\mathrm{R}} \propto\left(\lambda_{\mathrm{p}} \lambda_{\mathrm{s}}\right)^{-1}$, where $\lambda_{\mathrm{p}}$ is the pump wavelength and $\lambda_{\mathrm{s}}$ is the Stokes wavelength ${ }^{29}$. Similarly, we characterise the devices using a TM-polarised pump, where the light polarisation is along the $x$-axis. As shown in 
a

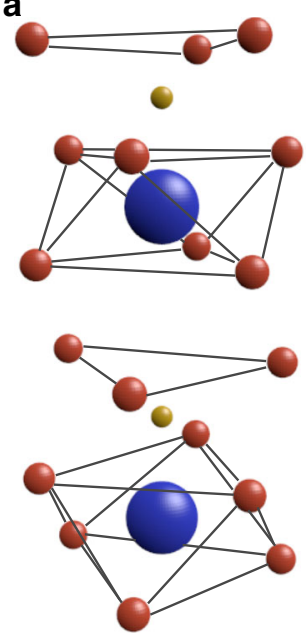

d

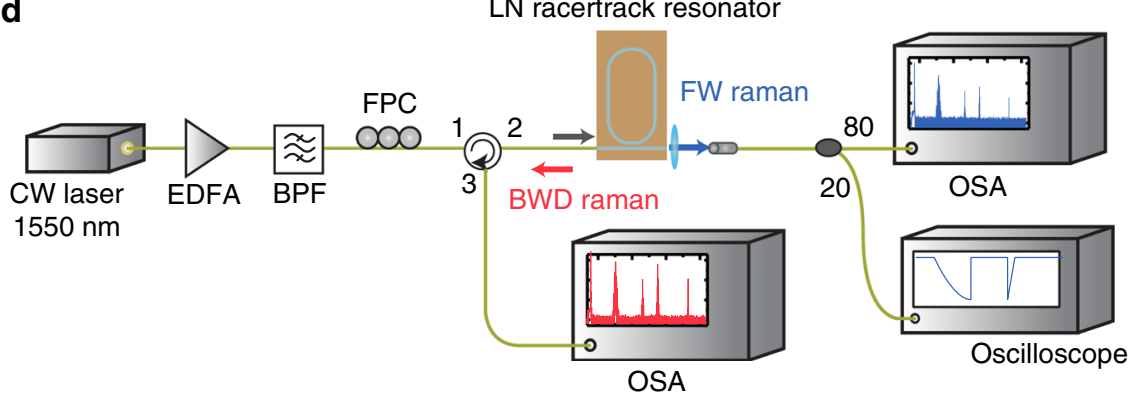

b

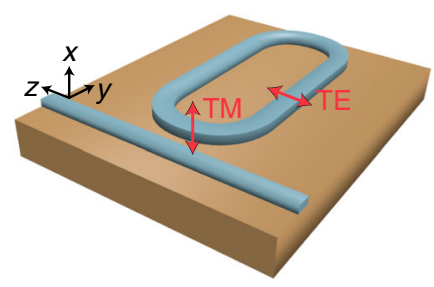

C
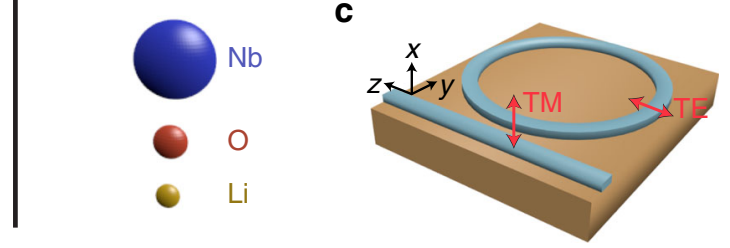


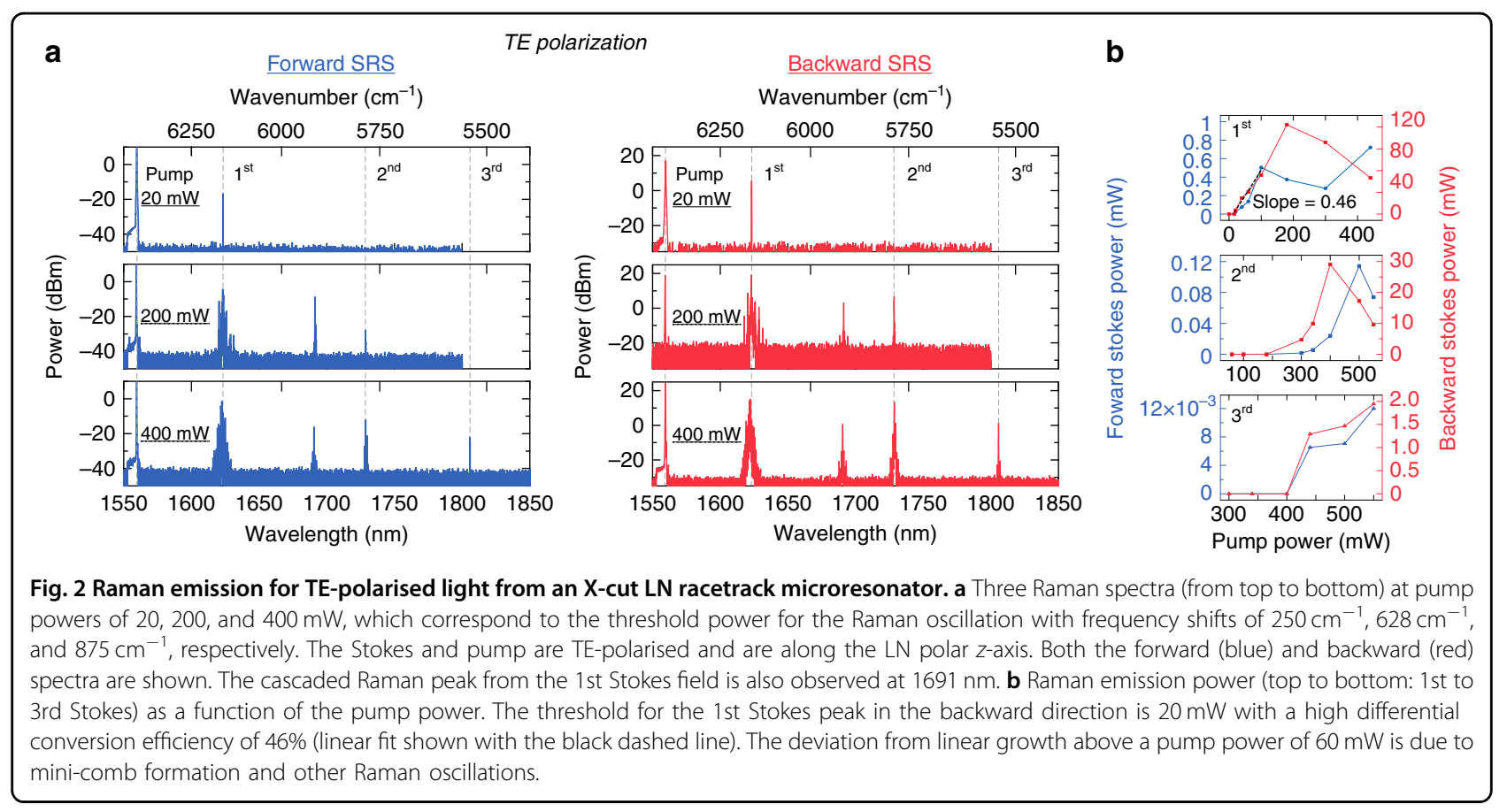

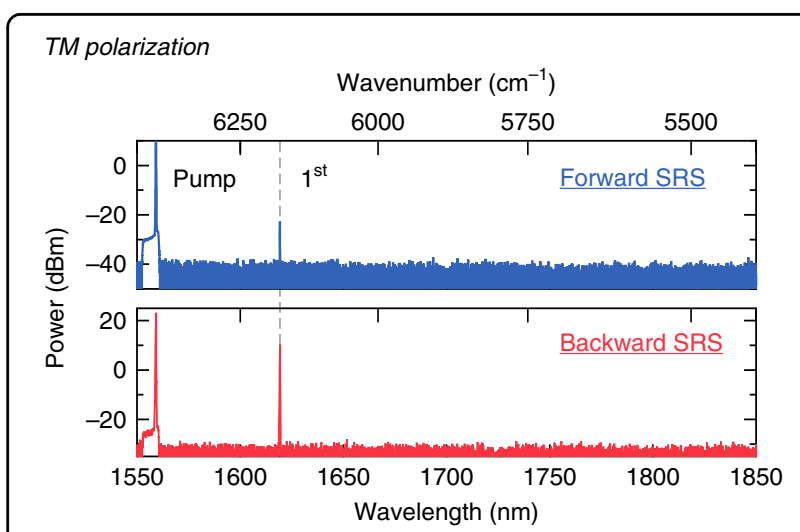

Fig. 3 Raman emission for the TM mode (the light polarisation is along the $x$-axis) at a frequency shift of $238 \mathrm{~cm}^{-1}$ at the threshold power of $340 \mathrm{~mW}$.

much weaker, and we do not observe a Raman oscillation at these pump powers; this is attributed to the fact that the Raman gain is less than the Kerr gain for the larger FSR. Figure $5 \mathrm{a}$ shows the generated spectra for an increased red-detuning of the pump. We observe the primary sidebands [State (i)], high-noise state [State (ii)], and multi-soliton state [State (iii)]. The RF spectra (Fig. 5b) show the reduction in the RF noise from State (ii) to State (iii), which strongly suggests the formation of a phase-locked state with narrow linewidth comb lines. By further detuning the pump laser, another phase-locked state with a more periodic spectral modulation is observed, which corresponds to the formation of a 5-soliton state within one cavity roundtrip (see Supplementary
Information). However, a single soliton is not achieved in the passive LN device. A fast switching approach, such as electrical tuning of the cavity resonance, could be utilised to possibly overcome the instabilities resulting from both the thermal-optic and photorefractive effects. Figure $5 c$ shows the transmission measurement of the resonator as the pump wavelength is swept through the resonance. The output is optically filtered using a long-pass filter with a cut-on wavelength of $1570 \mathrm{~nm}$. Figure $5 \mathrm{~d}$ shows a zoom-in of the dashed-rectangular region in Fig. $5 \mathrm{c}$ that indicates the 'soliton step' representing soliton formation ${ }^{48}$. The achieved mode-locked Kerr comb generation indicates that operating with the TM mode using an X-cut LN thin film allows for sufficient suppression of Raman effects. In contrast to the Z-cut LN thin film in ref. ${ }^{3}$, where the behaviour of self-starting or bidirectional tuning is observed as a result of the photorefractive effect, our microresonator is dominated by the thermo-optic effect. Moreover, our results do not indicate the occurrence of Raman self-frequency shifting observed by ref. ${ }^{3}$, which often occurs in an amorphous material. The difference in dynamics may be attributed to a thin film with different crystal orientations. We report the first demonstration of soliton mode-locking in X-cut LN microresonators compatible with active electrode control ${ }^{2,5}$.

\section{Discussion}

In conclusion, we achieve multi-wavelength Raman lasing on a monolithic LN chip and characterise the distinct Raman processes for different pump polarisations. All the Raman oscillations are dominant in the backward 

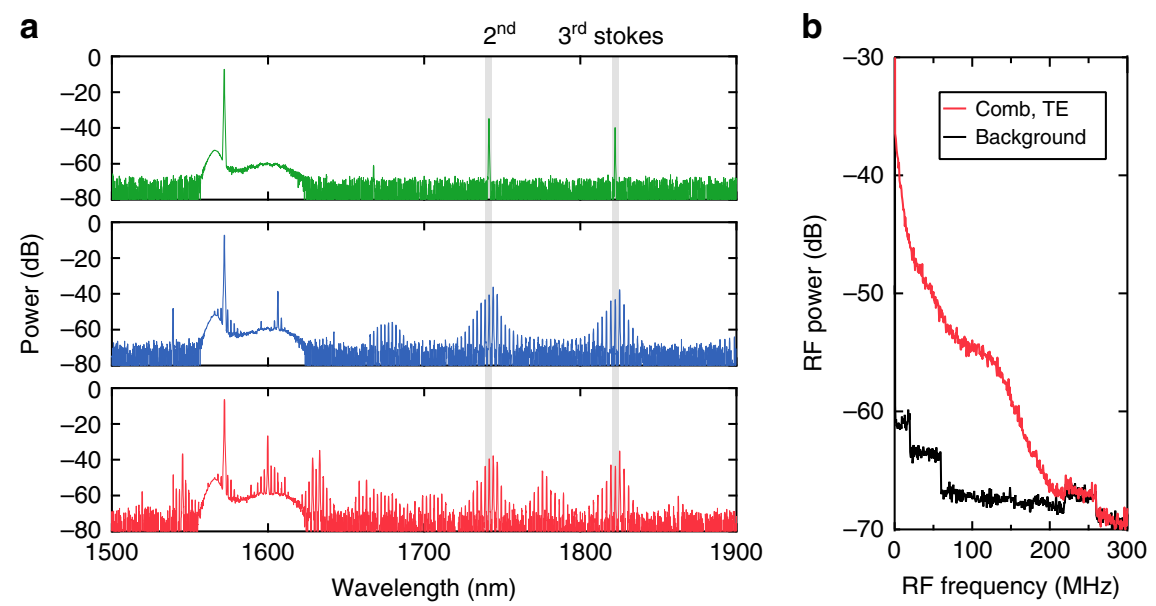

Fig. 4 Kerr frequency comb generation for TE-polarised light. a Raman and Kerr oscillations for the TE mode from an LN microresonator as the pump laser is tuned into the cavity resonance (top to bottom). $\mathbf{b}$ RF spectrum corresponding to the bottom spectra in $\mathbf{a}$.
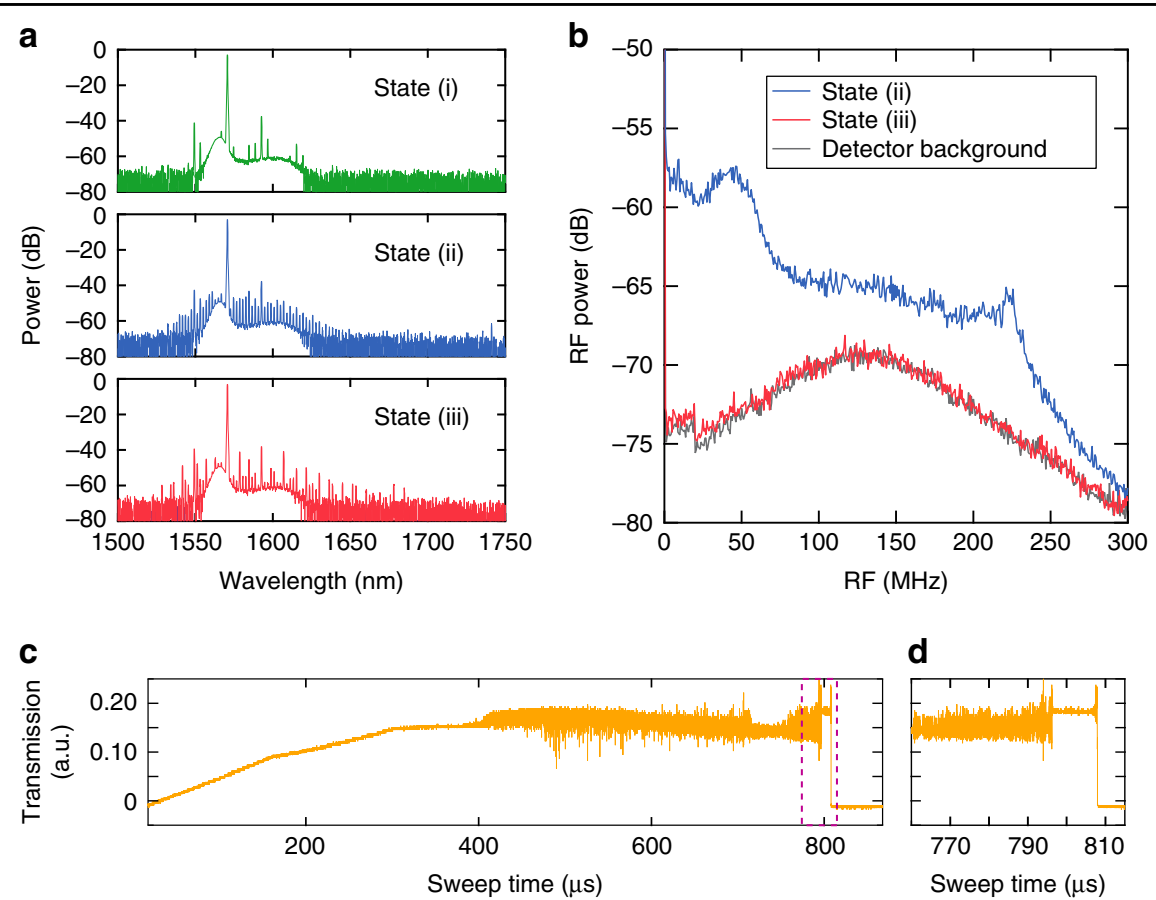

Fig. 5 Kerr frequency comb generation for TM-polarised light. a Kerr oscillation for the TM mode from an X-cut LN microresonator as the pump laser is red-detuned into the cavity resonance (top to bottom). b RF spectra corresponding to States (ii) and (iii) in $\mathbf{a}$. c Transmission measurement of the microresonator output above $1570 \mathrm{~nm}$, which excludes the pump wavelength. The laser is tuned to the resonance as the wavelength increases, dominated by the thermal-optic effect. The laser sweeping speed is $80 \mathrm{pm} / \mathrm{ms}$. $\mathbf{d}$ Zoom-in of the transmission trace in $\mathbf{c}$, which shows the onset of the soliton step.

direction with respect to the pump, and we report the highest pump-to-Stokes conversion efficiency of $46 \%$ for TE-polarised light. Operating in the normal GVD regime using a resonator with a higher $Q$ will enable a highly efficient Raman laser on an LN chip. Counter-propagating pump and Stokes fields might lead to richer nonlinear dynamics or functionalities such as symmetry breaking ${ }^{49}$, counter-propagating solitons and Stokes solitons ${ }^{50}$. In addition, we observe nontrivial interactions between the Raman effect and $x^{(3)}$-based FWM processes for Kerr comb formation. Although the strong contribution from the Raman effect impedes soliton formation for the TE polarisation, we demonstrate mode-locking for the TM polarisation through an optimisation of the cavity 
geometry. Alternatively, TE-polarised phase-locked combs could be achieved with the help of strong electrical driving $^{5,41}$. This work provides deep insight into the dynamics and effects of Raman scattering in the LNOI platform, which is critical for the design and development of chip-based nonlinear photonic devices.

\section{Materials and methods \\ Device parameters and fabrication}

For the Raman characterisation, we fabricate a racetrack microresonator from a commercial X-cut lithium niobate (LN) on an insulator wafer with a thin-film LN thickness of $800 \mathrm{~nm}$. The device is cladded with silicon oxide $750 \mathrm{~nm}$ in thickness with a top waveguide width of $1.2 \mu \mathrm{m}$ and a slab thickness of $350 \mathrm{~nm}$. The bending structure is based on Euler curves to avoid mode conversion between the transverse electric (TE) and transverse magnetic (TM) modes. The two straight sections are each $1.75 \mu \mathrm{m}$ in length along the $y$-axis. The coupling gap between the bus waveguide and microresonator is $0.75 \mu \mathrm{m}$, which leads to near-critical coupling for the TE modes and $45 \%$ transmission on resonance for the TM modes at $1550 \mathrm{~nm}$ (see Supplementary Information). The FSR is $30 \mathrm{GHz}$. For Kerr comb generation, the microring resonator is fabricated on a 600 -nm-thick thin-film LN wafer with a radius of $80 \mu \mathrm{m}$. The device is air-cladded with a slab thickness of $250 \mathrm{~nm}$ and a top waveguide width of $1.3 \mu \mathrm{m}$. The coupling gap is $830 \mathrm{~nm}$, which results in $50 \%$ transmission on resonance for the TE mode and $83 \%$ for the TM mode at $1550 \mathrm{~nm}$.

Electron-beam lithography (EBL, $125 \mathrm{keV}$ ) is used for patterning the waveguides and microcavities in hydrogen silsesquioxane resist (FOX). Then, the patterned LN wafer is etched using $\mathrm{Ar}^{+}$-based reactive ion etching by a userdefined etch depth. The $\mathrm{SiO}_{2}$ cladding is deposited by plasma-enhanced chemical vapour deposition. Finally, the chip facet is diced and polished, which typically results in a fibre-to-chip facet coupling loss of $7 \mathrm{~dB}$.

\section{Comb characterisation}

The group velocity dispersion (GVD) is simulated using commercial finite element analysis software (COMSOL) based on the fabricated device geometry. Anomalous GVD is achieved for both the TE and TM modes at telecommunication wavelengths (see Supplementary Information). A continuous-wave pump laser (Santec TSL510) at $1570 \mathrm{~nm}$ is amplified by an L-band erbium-doped fibre amplifier (EDFA, Manlight) and sent to the microring resonator using a lensed fibre after a polarisation controller. The tuning of the laser is controlled by a piezo controller. The output is collected by an aspheric objective followed by a fibre collimator. A 90:10 fibre beamsplitter is used to separate the output light into two arms. The $10 \%$ arm is sent to an optical spectrum analyser, and the $90 \%$ arm is sent through a home-built 4$f$ shaper (effectively a bandpass filter, $1575-1630 \mathrm{~nm}$ ) to filter the pump. The filtered comb spectrum is sent to a photodetector (Newport 1811, $125 \mathrm{MHz}$ ) and a real-time spectrum analyser (Tektronix RSA5126A). For Fig. 5c, a functional generator with a triangular function is sent to the piezo controller to scan the laser wavelength at $70 \mathrm{~Hz}$, and the filtered comb is collected by a photodetector (Thorlabs, PDA05CF2) followed by an oscilloscope (Tektronix DPO2024, $200 \mathrm{MHz}$ ).

\section{Acknowledgements}

The device fabrication is performed at the Harvard University Center for Nanoscale Systems (CNS), a member of the National Nanotechnology Coordinated Infrastructure Network (NNCl), which is supported by the National Science Foundation under NSF ECCS award No. 1541959. This work was supported by the National Science Foundation (NSF) (ECCS-1740296 E2CDA), Defense Advanced Research Projects Agency (DARPA) (W31P4Q-15-1-0013), and Air Force Office of Scientific Research (AFOSR) (FA9550-15-1-0303).

\section{Author details}

'Department John A. Paulson School of Engineering and Applied Sciences, Harvard University, Cambridge, MA 02138, USA. ${ }^{2}$ Department of Applied Physics and Applied Mathematics, Columbia University, New York, NY 10027, USA. ${ }^{3}$ Department of Electronic Engineering \& State Key Laboratory of Terahertz and Millimeter Waves, City University of Hong Kong, Kowloon, Hong Kong, China. ${ }^{4}$ HyperLight Corporation, 501 Massachusetts Avenue, Cambridge, MA 02139, USA

\section{Author contributions}

M.Y. and Y.O. prepared the manuscript in discussion with all authors. M.Y. and Y.O. designed and performed the experiments. M.Y., Y.O., C.W. and M.Z. analysed the data. R.C. and C.W. fabricated the devices. M.L. and A.L.G. supervised the project.

\section{Data availability}

The data that support the plots within this paper and other findings of this study are available from the corresponding author upon reasonable request.

Conflict of interest

The authors declare that they have no conflict of interest.

Supplementary information is available for this paper at https://doi.org/ 10.1038/s41377-020-0246-7.

Received: 27 June 2019 Revised: 30 December 2019 Accepted: 6 January 2020

Published online: 20 January 2020

\footnotetext{
References

1. Zhang, M. et al. Monolithic ultra-high-Q lithium niobate microring resonator. Optica 4, 1536-1537 (2017).

2. Wang, C. et al. Monolithic lithium niobate photonic circuits for Kerr frequency comb generation and modulation. Nat. Commun. 10, 978 (2019).

3. He, Y. et al. Self-starting bi-chromatic $\mathrm{LiNbO}_{3}$ soliton microcomb. Optica 6 , 1138-1144 (2019).

4. Gong, Z. et al. Soliton microcomb generation at $2 \mu \mathrm{m}$ in z-cut lithium niobate microring resonators. Opt. Lett. 44, 3182-3185 (2019).

5. Zhang, $\mathrm{M}$. et al. Broadband electro-optic frequency comb generation in a lithium niobate microring resonator. Nature 568, 373-377 (2019).

6. Luo, R. et al. On-chip second-harmonic generation and broadband parametric down-conversion in a lithium niobate microresonator. Opt. Express 25, 24531-24539 (2017)

7. Luo, R. et al. Highly tunable efficient second-harmonic generation in a lithium niobate nanophotonic waveguide. Optica 5, 1006-1011 (2018).
} 
8. Wang, C. et al. Ultrahigh-efficiency wavelength conversion in nanophotonic periodically poled lithium niobate waveguides. Optica 5, 1438-1441 (2018).

9. $\mathrm{Yu}, \mathrm{M}$. J. et al. Coherent two-octave-spanning supercontinuum generation in lithium-niobate waveguides. Opt. Lett. 44, 1222-1225 (2019).

10. Lu, J. J. et al. Octave-spanning supercontinuum generation in nanoscale lithium niobate waveguides. Opt. Lett. 44, 1492-1495 (2019).

11. Wolf, R. et al. Cascaded second-order optical nonlinearities in on-chip micro rings. Opt. Express 25, 29927-29933 (2017).

12. Guarino, A. et al. Electro-optically tunable microring resonators in lithium niobate. Nat. Photonics 1, 407-410 (2007).

13. Rao, A. \& Fathpour, S. Heterogeneous thin-film lithium niobate integrated photonics for electrooptics and nonlinear optics. IEEE J. Sel. Top. Quantum Electron. 24, 8200912 (2018).

14. Boes, A. et al. Status and potential of lithium niobate on insulator (LNOI) for photonic integrated circuits. Laser Photonics Rev. 12, 1700256 (2018).

15. Desiatov, B. et al. Ultra-low-loss integrated visible photonics using thin-film lithium niobate. Optica 6, 380-384 (2019).

16. Rueda, A. et al. Resonant electro-optic frequency comb. Nature $\mathbf{5 6 8}$, 378-381 (2019).

17. Barker, A. S. Jr \& Loudon, R. Dielectric properties and optical phonons in LiNbO3. Phys. Rev. 158, 433-445 (1967).

18. Schaufele, R. F. \& Weber, M. J. Raman scattering by lithium niobate. Phys. Rev. 152, 705-708 (1966).

19. Kaminow, I. P. \& Johnston, W. D. Jr Quantitative determination of sources of the electro-optic effect in $\mathrm{LiNbO}_{3}$ and $\mathrm{LiTaO}_{3}$. Phys. Rev. 160, 519-522 (1967).

20. Caciuc, V., Postnikov, A. V. \& Borstel, G. Ab initio structure and zone-center phonons in LiNbO3. Phys. Rev. B 61, 8806-8813 (2000).

21. Boyd, R. W. Nonlinear Optics. 3rd edn. (Academic Press, Inc, Orlando, FL, USA, Academic Press, Inc, 2008)

22. Johnston, W. D. Jr, Kaminow, I. P. \& Bergman, J. G. Jr Stimulated Raman gain coefficients for Li6NbO3, Ba2NaNb5O15, and other materials. Appl. Phys. Lett. 13, 190-193 (1968).

23. Bache, M. \& Schiek, R. Review of measurements of Kerr nonlinearities in lithium niobate: the role of the delayed Raman response. Preprint at https://arxiv.org/ abs/1211.1721 (2012).

24. Leidinger, M. et al. Strong forward-backward asymmetry of stimulated Raman scattering in lithium-niobate-based whispering gallery resonators. Opt. Lett. $\mathbf{4 1}$ 2823-2826 (2016)

25. Wu, R. B. et al. Lithium niobate micro-disk resonators of quality factors above 107. Opt. Lett. 43, 4116-4119 (2018).

26. Maleki, L. et al. Whispering gallery mode lithium niobate microresonators for photonics applications. In Proceedings of Enabling Photonic Technologies for Aerospace Applications V. (SPIE, Orlando, Florida, USA, 2003).

27. Rong, H. S. et al. A cascaded silicon Raman laser. Nat. Photonics 2, 170-174 (2008).

28. Latawiec, P. et al. On-chip diamond Raman laser. Optica 2, 924-928 (2015).

29. Spillane, S. M., Kippenberg, T. J. \& Vahala, K. J. Ultralow-threshold Raman laser using a spherical dielectric microcavity. Nature 415, 621-623 (2002).
30. Kippenberg, T. J. et al. Ultralow-threshold microcavity Raman laser on a microelectronic chip. Opt. Lett. 29, 1224-1226 (2004).

31. Grudinin, I. S. \& Maleki, L. Ultralow-threshold Raman lasing with $\mathrm{CaF}_{2}$ resonators. Opt. Lett. 32, 166-168 (2007).

32. Vanier, F. et al. Raman lasing in As $2 S 3$ high-Q whispering gallery mode resonators. Opt. Lett. 38, 4966-4969 (2013).

33. Liu, X. W. et al. Integrated continuous-wave aluminum nitride Raman laser. Optica 4, 893-896 (2017).

34. Latawiec, P. et al. Integrated diamond Raman laser pumped in the near-visible. Opt. Lett. 43, 318-321 (2018).

35. Chembo, Y. K., Grudinin, I. S. \& Yu, N. Spatiotemporal dynamics of Kerr-Raman optical frequency combs. Phys. Rev. A 92, 043818 (2015).

36. Okawachi, Y. et al. Competition between Raman and Kerr effects in microresonator comb generation. Opt. Lett. 42, 2786-2789 (2017).

37. Liu, X. W. et al. Integrated high-Q crystalline AIN microresonators for broadband Kerr and Raman frequency combs. ACS Photonics 5, 1943-1950 (2018).

38. Cherenkov, A. V. et al. Raman-Kerr frequency combs in microresonators with normal dispersion. Opt. Express 25, 31148-31158 (2017).

39. Fujii, S. et al. Transition between Kerr comb and stimulated Raman comb in a silica whispering gallery mode microcavity. J. Optical Soc. Am. B 35, 100-106 (2018).

40. Møller, U. \& Bang, O. Intensity noise in normal-pumped picosecond supercontinuum generation, where higher-order Raman lines cross into anomalous dispersion regime. Electron. Lett. 49, 63-65 (2013).

41. Zhang, M. et al. Microresonator frequency comb generation with simultaneous Kerr and electrooptic nonlinearities. Proceedings of 2019 Conference on Lasers and Electro-Optics, FF2D (Optical Society of America, San Jose, 2019).

42. Hansson, T., Modotto, D. \& Wabnitz, S. Mid-infrared soliton and Raman frequency comb generation in silicon microrings. Opt. Lett. 39, 6747-6750 (2014).

43. Jestin, Y. et al. Improving resonant photonics devices with sol-gel coatings Proceedings of Laser Resonators and Beam Control XI (SPIE, San Jose, California, USA, 2009).

44. Puthoff, H. E. et al. Near-forward Raman scattering in $\mathrm{LiNbO}_{3}$. J. Appl. Phys. 39 2144-2146 (1968)

45. Krause, M., Renner, H. \& Brinkmeyer, E. Strong enhancement of Ramaninduced nonreciprocity in silicon waveguides by alignment with the crystallographic axes. Appl. Phys. Lett. 95, 261111 (2009).

46. Warrier, A. M. et al. Multiwavelength ultrafast $\mathrm{LiNbO}_{3}$ Raman laser. Opt. Express 23, 25582-25587 (2015).

47. Joshi, C. et al. Thermally controlled comb generation and soliton modelocking in microresonators. Opt. Lett. 41, 2565-2568 (2016).

48. Herr, T. et al. Temporal solitons in optical microresonators. Nat. Photonics $\mathbf{8}$ 145-152 (2014)

49. Del Bino, L. et al. Symmetry breaking of counter-propagating light in a nonlinear resonator. Sci. Rep. 7, 43142 (2017).

50. Yang, Q. F. et al. Stokes solitons in optical microcavities. Nat. Phys. 13, 53-57 (2017). 